\title{
Les soignants et la mort - Multiples interrogations
}

Jean Martin

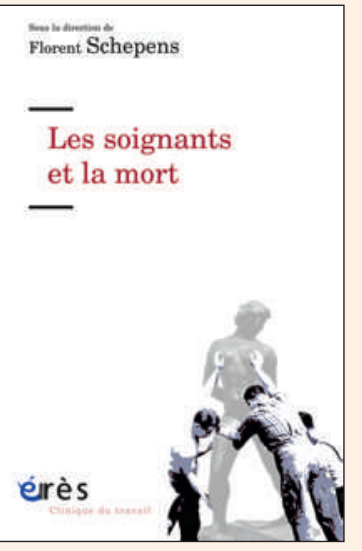

Florent Schepens (éd.) Les soignants et la mort Toulouse: Editions érès; 2013. 262 pages. 15 Euros. ISBN 978-2-7492-3616-2

jean.martin[at]saez.ch
Dans un billet récent [1], j'ai évoqué deux contributions de cet ouvrage publié dans une collection «Clinique du travail», sous la direction d'un sociologue de l'Université de Bourgogne. Il rassemble les contributions de 19 auteurs: 8 sociologues, 3 philosophes, 5 soignants dont deux médecins, trois autres. Mais le professionnel de la santé ne doit pas y craindre une «surcharge» de sciences humaines. Le tout est substantiel, illustrant une approche réfléchie de la souffrance en fin de vie; en prise sur l'époque et les évolutions (judicieuses) intervenues au cours des décennies récentes. Ceci alors que les pratiques restent encore trop souvent, au jour le jour, marquées par des résistances plus vraiment acceptables à traiter suffisamment la douleur et à parler de la fin de sa vie avec le malade.

Quatre parties, avec pour thèmes: 1) différentes définitions de la mort: articles sur la problématique en service de réanimation, les états végétatifs chroniques avec une discussion des rapports avec les proches, et un historique de l'évolution du régime anthropologique de la mort; 2 ) «travailler les corps»: qu'il s'agisse de dépouilles (activité du personnel «aide d'anatomie», dissection par les étudiants), de chirurgie où l'humour noir sert de moyen de distanciation, d'une description des politiques d'humanisation de la mort à l'hôpital; 3) la relation comme pratique soignante: quatre travaux sur la fin de vie, sur les rapports (en oncologie) entre prise en charge à visée curative et soins palliatifs, le rôle des médecins généralistes dans les fins de vie à domicile, la mort «ordinaire» dans les établissements pour personnes âgées dépendantes; 4) l'élaboration de la mort et du mourir: avec un texte d'un philosophe, deux de sociologues (l'un sur les «morts difficiles» que sont celles d'adolescents/jeunes adultes ou par suicide), et un sur des études de la division morale du travail à l'hôpital et la possible émergence, chez les soignants, de stratégies défensives.

\section{L'époque de la mort-tabou et de la non-vérité}

Cette époque n'est pas si loin (elle se prolonge ici et là). «Le souci du médecin de cacher la proximité de la mort est la logique qui prédomine jusqu'aux années 1970 [...] Ce silence face au mourant est même considéré comme un soutien efficace pour le malade. Le Professeur Jean Hamburger (néphrologue connu, années 1960-1970) dans une note pour ses étudiants: «Que faire si votre conviction est que la maladie est mortelle? C'est ici l'exception dont je parlais plus haut: il faut mentir, mentir dans tous les cas; cette exigence est purement et simplement thérapeutique [...]» Attitude aujourd'hui considérée comme tout à fait inadaptée qui a pour résultat de «voler sa mort» au malade. Au reste, le fait est que l'évolution vers le refus du mensonge et vers le dialogue à propos de l'inéluctabilité d'une issue funeste reste incomplète (les régions anglo-saxonnes et nordiques étant plus ouvertes voire exigeantes en matière d'annonce véridique et spontanée au malade et, s'il y consent, à ses proches). Grandes différences culturelles: je me souviens, dans un congrès de bioéthique, d'un médecin canadien disant «je découvre ici au Japon que c'est une faute professionnelle de dire à un patient qu'il a un cancer alors que, dans mon pays, ne pas le dire mettrait en danger mon autorisation de pratiquer».

\section{Les soignants et la mort}

Quelques citations: «Ces dernières décennies, le regard médical sur la fin de vie et la mort, qui a profondément changé, a fait repenser la prise en charge des malades mourants. Avec les soins palliatifs, on se situe dans une logique qui accorde une place centrale à la parole du malade et à son histoire», rappelle M. Castra. Qui ajoute: «La relation au patient est ici une forme de compétence étroitement liée à une nouvelle conception de l'éthique de fin de vie.»

«Si pendant des années la réanimation a effectivement occulté le patient-sujet, exclu les familles et caché la mort, elle est aujourd'hui une spécialité qui a beaucoup fait évoluer ses pratiques [...] Le soignant peut cependant osciller entre deux attitudes: prendre en charge un patient objet de soins ou prendre en charge un patient-sujet.» "C'est lorsque le patient entre en 'fin de vie` qu'une brèche s'ouvre pour les émotions et l'expression du vécu de chacun [...] Il s'opère un véritable basculement: le patient est l'objet d'une requalification - il (re)devient une personne» (N. Kentish-Barnes et J. Valy).

«L'importance accordée à la relation dans le travail palliatif est un des points d'appui essentiels, avec les morphines et les traitements des symptômes, légitimant cette approche» (M. Castra). Tiré d'une étude: «De manière générale, les médecins spécialistes font peu référence à la gravité de la situation de la personne cancéreuse. Ce qui se produit dans l'espace domestique (en cas de chimiothérapie orale à domicile) n'intéresse le spécialiste que si cela a un impact direct sur la prise du médicament. 
L'enjeu central demeure l'observance» (R. - A. Foley et al.).

«Faire du doute une ressource: le doute, l'incertitude, est un état normal quand on prend soin de personnes mourantes et les certitudes y sont décrites comme dangereuses» (dit F. Schepens des enseignements d'une recherche).

\section{La mort est l'affaire de tous}

C'est un des sous-titres de la conclusion du livre par le palliatologue Régis Aubry, de Besançon. A propos des professionnels: «Or, le soignant est placé souvent face à la question des limites: celles de la vie, celle du savoir, ainsi que les siennes propres.» «Que peut faire un professionnel en situation d'incertitude. Bien sûr, il va appliquer ce qu'il a appris à faire; il va traiter la douleur, les symptômes d'inconfort. Puis il va se demander ce que soigner veut dire lorsque guérir est impossible. Il va devoir entendre l'indicible, il n'abandonnera pas le patient.» Au reste, la mort ne doit pas être laissée aux seuls professionnels de santé. Rendre la mort à la société et à ce qui se joue en son sein. "Chaque citoyen a été, est ou sera un jour confronté aux nombreuses questions posées par la finitude et la mort. Chacun éprouvera ce que la solidarité veut dire face à la souffrance, à l'altérité et à la vulnérabilité.»

1 Martin J. La moins mauvaise solution est la meilleure. (Das kleinere Übel ist immer noch die bessere Lösung). Bull Méd Suisses. 2013;94(48):1840 . 